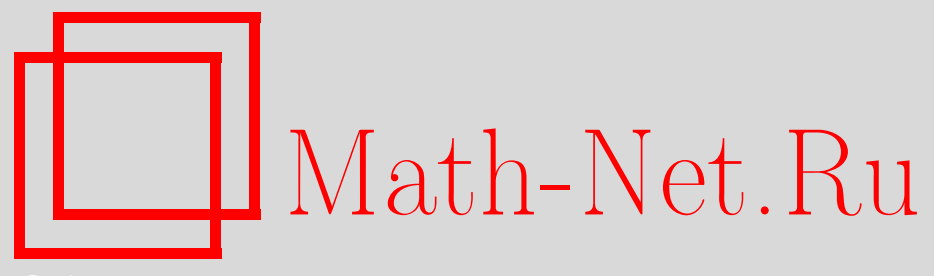

Л. А. Бекларян, Группы гомеоморфизмов прямой. Критерии существования инвариантной и проективно инвариантной мер в терминах коммутанта, Матем. сб., 2014, том 205, номер 12, 63-84

DOI: https://doi.org/10.4213/sm8306

Использование Общероссийского математического портала Math-Net.Ru подразумевает, что вы прочитали и согласны с пользовательским соглашением http://www . mathnet.ru/rus/agreement

Параметры загрузки:

IP : 44.207 .124 .84

26 апреля 2023 г., $17: 32: 01$

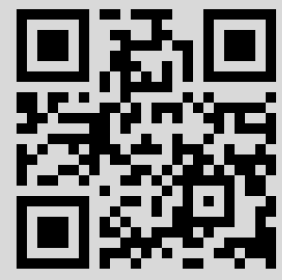




\title{
Л. А. Бекларян \\ Группы гомеоморфизмов прямой. Критерии существования инвариантной и проективно инвариантной мер в терминах коммутанта
}

\begin{abstract}
В работе для группы $G$ гомеоморфизмов прямой получены критерии существования инвариантной и проективно инвариантной мер, сформулированные в терминах коммутанта $[G, G]$. Для частного, но очень важного случая групп гомеоморфизмов прямой со свободно действующим элементом удается получить критерий существования проективно инвариантной меры в виде условия отсутствия специальной подгруппы с двумя образующими, в которой одним из образующих элементов является свободно действующий элемент.

Библиография: 20 названий.
\end{abstract}

Ключевые слова: группы гомеоморфизмов прямой (окружности), инвариантная мера, проективно инвариантные меры.

DOI: $10.4213 / \mathrm{sm} 8306$

\section{Введение}

Группы гомеоморфизмов прямой (окружности) возникают при изучении различных задач геометрии, групп квазиконформных отображений, функционально-дифференциальных уравнений, теории волн, вариационного исчисления и т.д. [1]-[12]. Их можно классифицировать на основе различных характеристик. Такими характеристиками могут служить свойство аменабельности, функция роста группы (для конечно порожденных групп), структура орбит (для групп гомеоморфизмов локально компактного пространства) и т.д. Для групп гомеоморфизмов прямой наиболее детальная классификация основана на характеристиках серии метрических инвариантов [13]-[17]. Поэтому важны критерии существования таких инвариантов. Они могут быть сформулированы в различных терминах, что позволяет детализировать классификационную схему в этих терминах. Критерий существования инвариантной меры впервые был получен Plante [18] для конечно порожденных групп гомеоморфизмов в терминах асимптотических свойств орбит. К сожалению, такой критерий не позволяет распространить его на группы, не являющиеся конечно порожденными. В [13] удалось получить критерий существования инвариантной меры для произвольной группы гомеоморфизмов прямой, сформулированный в терминах топологической характеристики исходной группы. Такой критерий оказался весьма

Работа выполнена при поддержке Российского фонда фундаментальных исследований (грант № 12-01-00768-а) и Программы поддержки ведущих научных школ РФ (грант № НШ-5998.2012.1). 
продуктивным, так как он допускал переформулировки в терминах различных характеристик исходной группы. Точно так же были получены критерии существования более общих метрических инвариантов. Так в [14], [15] в терминах свойств канонических подгрупп исходной группы гомеоморфизмов прямой получен критерий существования проективно инвариантной меры. В [16] в терминах топологических свойств исходной группы гомеоморфизмов прямой получен признак существования $\omega$-проективно инвариантной меры. В [16], [17] для групп гомеоморфизмов прямой получен критерий существования инвариантной меры, который формулируется на основе комбинаторных характеристик группы (отсутствие свободных подполугрупп с двумя образующими для некоторой канонической факторгруппы). Там же для специальных групп, содержащих нормальную подгруппу с инвариантной мерой и свободно действующим элементом, получен критерий существования проективно инвариантной меры на основе комбинаторных характеристик группы (отсутствие свободных подгрупп с двумя образующими для некоторой канонической факторгруппы). В частности, в [19] для групп гомеоморфизмов окружности получен критерий существования инвариантной меры, который также формулируется на основе комбинаторных характеристик группы (отсутствие свободных подгрупп с двумя образующими для некоторой канонической факторгруппы). Вместе с тем вопрос формулировки критериев существования метрических инвариантов в новых терминах остается актуальным.

В представленной работе для группы $G$ гомеоморфизмов прямой, сохраняющих ориентацию, будут сформулированы критерии существования инвариантной (теорема 2.1) и проективно инвариантной (теорема 2.2) мер в терминах взаиморасположения коммутанта $[G, G]$ и выделенных канонических подгрупп исходной группы $G$. Будет дана эквивалентная переформулировка приводимого критерия существования проективно инвариантной меры через свойства графиков гомеоморфизмов из коммутанта $[G, G]$ (лемма 2.1), а также исходной группы $G$ (лемма 2.2). Для частного, но очень важного случая групп гомеоморфизмов прямой со свободно действующим элементом удается получить критерий существования проективно инвариантной меры в иных терминах (теорема 3.1). Такой критерий формулируется в виде условия отсутствия специальной подгруппы с двумя образующими, в которой одним из образующих элементов является свободно действующий элемент.

\section{§ 1. Некоторые определения и предварительные результаты}

Для замкнутости изложения приведем ряд определений, а также предварительных результатов. В приведенных результатах будут даны две ссылки. Первая ссылка на работу, где результат был опубликован впервые, вторая ссылка на обзорную статью, в которой собраны все эти результаты.

В дальнейшем через $\mathrm{Homeo}_{+}(\mathbb{X}), \mathbb{X}=\mathbb{R}, \mathbb{S}^{1}$, будем обозначать группу всех гомеоморфизмов $\mathbb{X}$, сохраняющих ориентацию. Определим важное каноническое подмножество $G^{S}$ группы $G$ как объединение стабилизаторов

$$
G^{S}=\bigcup_{t \in \mathbb{X}} \operatorname{St}_{G}(t)
$$


Множество $G^{S}$ может и не быть группой. Очевидны вложения

$$
G^{S} \subseteq\left\langle G^{S}\right\rangle \subseteq G
$$

Оказывается, такая цепочка вложений характеризуется экстремальным свойством, описанным в следующей ниже лемме.

Лемма 1.1 (см. [13], [17]). Пусть $G \subseteq$ Homeo+ $_{+}(\mathbb{R})$. Тогда или $G^{S}=\left\langle G^{S}\right\rangle$, или $\left\langle G^{S}\right\rangle=G$.

Альтернатива, сформулированная в лемме, является нестрогой, так как существуют группы, для которых $G^{S}=G$. Приведенная лемма лежит в основе важной теоремы о факторгруппе.

TEOPEмA 1.1 (см. [13], [17]). Пусть $G \subseteq$ Homeo $_{+}(\mathbb{X})$. Тогда факторгруnпа $G /\left\langle G^{S}\right\rangle$ коммутативна и изоморфна некоторой подгруппе аддитивной груп$n b \iota \mathbb{X}$.

ЗАмечание 1.1. Пусть $G \subseteq$ Homeо $_{+}(\mathbb{X})$. Если $G /\left\langle G^{S}\right\rangle \neq\langle e\rangle$, то в группе $G$ существует свободно действующий элемент.

Теорема о структуре факторгруппы является определяющей при исследовании групп гомеоморфизмов прямой (окружности). Вместе с тем такая характеристика, как факторгруппа $G /\left\langle G^{S}\right\rangle$, не может быть универсальным инструментом при классификации групп гомеоморфизмов прямой, так как она является нетривиальной только лишь для групп с инвариантной мерой (см. теорему 1.4). В частности, для разрешимой группы $G=\langle t+1,2 t\rangle$ с двумя образующими факторгруппа $G /\left\langle G^{S}\right\rangle$ тривиальная и не несет в себе никакой новой информации об исходной группе $G$.

1.1. Минимальные множества. Для группы $G \subseteq \mathrm{Homeo}_{+}(\mathbb{X})$ важной топологической характеристикой является минимальное множество.

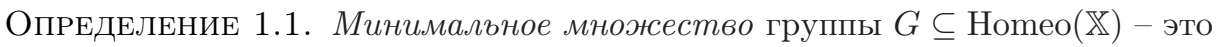
замкнутое $G$-инвариантное подмножество $\mathbb{X}$, не содержащее собственных замкнутых $G$-инвариантных подмножеств. Если не существует непустого минимального множества, то по определению будем полагать, что оно пустое.

Важность минимальных множеств определяется тем, что в случае их "нетривиальности" орбиты точек обладают некоторыми каноническими свойствами, а сами минимальные множества определяют носители метрических инвариантов.

Другой важнейшей топологической характеристикой является множество

$$
\text { Fix } G^{S}=\left\{t \in \mathbb{X}: \forall g \in G^{S} g(t)=t\right\} .
$$

Tеорема 1.2 (см. [15], [17]). Пусть $G \subseteq \mathrm{Homeo+}_{+}(\mathbb{R})$. Тогда справедливо одно из следующих взаимоисключающих утверждений:

а) любое минимальное множество дискретно и принадлежит множеcтву Fix $G^{S}$, а множество Fix $G^{S}$ coстоит из обгединения некоторой совокупности минималъных множеств $E_{\alpha}, \alpha \in \mathcal{A}$, m.e. Fix $G^{S}=$ $\bigcup_{\alpha \in \mathcal{A}} E_{\alpha}$ 
б) минимальное множество является совершенным нигде не плотным подмножеством $\mathbb{R}$. В этом случае оно является единственным минимальным множеством и содержится в замыкании орбиты $\overline{G(t)}$ произвольной точки $t \in \mathbb{R}$;

в) минимальное множество совпадает с $\mathbb{R}$;

г) минимальное множество пустое.

Недискретное минимальное множество группы $G$ (оно единственное) будем обозначать через $E(G)$.

Приведем признак существования непустого минимального множества. Определим подмножество

$$
G_{\infty}^{S}=\left\{g \in G^{S}: \sup \{t: g(t)=t\}=+\infty, \inf \{t: g(t)=t\}=-\infty\right\} .
$$

ПрЕДЛОЖЕНИЕ 1.1 (см. [15], [16]). Пусть $G \subseteq$ Нотео+ $_{+}(\mathbb{R})$ и выполняется хотя бъ одно из условий:

а) $G$ - конечно порожденная группа;

б) $\operatorname{Fix} G^{S} \neq \varnothing$;

в) $G \neq G_{\infty}^{S}$.

Тогда существует непустое минимальное множество.

Определим одну важную каноническую подгруппу исходной группы, связанную с минимальным множеством.

ОПРЕДЕЛЕНИЕ 1.2. Для группы $G \subseteq \operatorname{Homeo}_{+}(\mathbb{X})$ нормалъная подгрупnа $H_{G}$ определяется следующим образом:

1) если минимальное множество непусто и недискретно, то положим

$$
H_{G}=\{h \in G: E(G) \subseteq \operatorname{Fix}\langle h\rangle\} ;
$$

2) если минимальное множество непусто и дискретно, то положим $H_{G}=G^{S}$ (из дискретности минимального множества следует непустота множества Fix $G^{S}$, из непустоты Fix $G^{S}$ следует, что $G^{S}$ является нормальной подгруппой);

3) если минимальное множество пусто, то положим $H_{G}=\langle e\rangle$.

Заметим, что если минимальное множество совпадает со всей прямой, то $H_{G}=\langle e\rangle$.

\section{2. Инвариантные меры.}

ОПредЕЛЕНиЕ 1.3. Борелевская мера $\mu$, конечная на компактах, называется инвариантной относительно группы $G \subseteq \mathrm{Homeo}_{+}(X)$, если для любого элемента $g \in G$ и любого борелевского подмножества $B \subseteq \mathbb{R}$ справедливо условие $\mu\left(g^{-1}(B)\right)=\mu(B)$.

Сформулируем критерий существования инвариантной меры в терминах топологических характеристик.

Теорема 1.3 (см. [13], [17]). Пусть $G \subseteq$ Нотео $_{+}(\mathbb{X})$. Тогда следующие утверждения эквивалентны: 
1) существует борелевская (вероятностная в случае $\mathbb{X}=\mathbb{S}^{1}$ ) мера $\mu$, конечная на компактах и инвариантная относительно группь $G$;

2) множество Fix $G^{S}$ непусто.

Впервые критерий существования инвариантной меры для конечно порожденной группы гомеоморфизмов прямой (окружности) сформулировал Plante [18]: для конечно порожденной группљ $G \subseteq \mathrm{Homeo+}_{+}(\mathbb{X})$ инвариантная борелевская мера, конечная на компактах, существует тогда и только тогда, когда существует точка $t \in \mathbb{X}$ с субэкспоненциальным ростом орбиты, т.е. $\liminf _{n \rightarrow 0}\left(\ln \left|G^{n}(t)\right|\right) / n=0$.

Здесь в конечно порожденной группе $G=\left\langle g_{1}, \ldots, g_{r}\right\rangle$ слова длины не более чем $n$, где $n=1,2, \ldots$, определяются как множество

$$
\begin{aligned}
G^{n}=\left\{g: g \in G, g=g_{i_{1}}^{\varepsilon_{1}}, \ldots, g_{i_{l}}^{\varepsilon_{l}}, l \leqslant n, \varepsilon_{p}= \pm 1, p=1, \ldots, l, i_{p} \in\{1, \ldots, r\}\right. & \\
& \left.g_{i_{p}}^{\varepsilon_{p}} \neq g_{i_{p+1}}^{-\varepsilon_{p+1}}, p=1, \ldots,(l-1), l>1\right\} .
\end{aligned}
$$

Критерий Plante хоть и формулируется в асимптотической форме, но в действительности носит топологический характер и означает непустоту носителя инвариантной меры. Критерий Plante становится понятным с учетом теорем $1.1,1.3$ и того, что при $\operatorname{Fix} G^{S} \neq \varnothing$ множество $G^{S}$ образует группу. K сожалению, критерий Plante не может быть распространен на группы, не являющиеся конечно порожденными.

Приведем признак существования инвариантной меры в терминах факторгруппы $G /\left\langle G^{S}\right\rangle$.

TeOpema 1.4 (см. [13], [17]). Пусть $G \subseteq$ Homeo+ $_{+}(\mathbb{X})$. Если факторгруnnа $G /\left\langle G^{S}\right\rangle$ нетривиальная, то существует борелевская мера $\mu$, конечная на компактах и инвариантная относительно группы $G$.

ЗАмЕчАНИЕ 1.2. В силу предложения 1.1 и теоремы 1.3 для группы $G \subseteq$ $\mathrm{Homeo}_{+}(\mathbb{R})$ из существования инвариантной меры следует, что минимальное множество непусто.

ЗАмечАниЕ 1.3. Пусть $G \subseteq$ Homeo $_{+}(\mathbb{X})$ и для группы $G$ минимальное множество непусто. Очевидно, что для группы $G$ выполняется включение $H_{G} \subseteq G^{S}$. Если существует борелевская мера $\mu$, конечная на компактах и инвариантная относительно группы $G$, то имеет место равенство $H_{G}=G^{S}$. Если выполняется условие $H_{G}=G^{S}$, то для группы $G$ существует инвариантная мера.

ЗАмечАниЕ 1.4. Если для группы $G \subseteq \mathrm{Homeo}_{+}(\mathbb{X})$ минимальное множество дискретное, то существует борелевская мера $\mu$, конечная на компактах и инвариантная относительно группы $G$.

Мы уже отмечали, что такая характеристика, как факторгруппа $G /\left\langle G^{S}\right\rangle$, не может быть универсальным инструментом при классификации групп гомеоморфизмов прямой, так как она оказывается нетривиальной только лишь для групп с инвариантной мерой. С другой стороны, топологическая характеристика $\operatorname{Fix} G^{S} \neq \varnothing$ из теоремы 1.3 также не дает никакой информации о группах, не удовлетворяющих этим критериям (признакам). Этот недостаток 
может быть преодолен за счет переформулировки отмеченных критериев в виде альтернатив.

В терминах комбинаторных характеристик сформулируем критерий существования инвариантной борелевской меры на окружности в виде строгой альтернативы, являющейся неулучшаемым усилением теоремы (критерия) Боголюбова-Крылова-Дейя о существовании инвариантной меры для аменабельной группы, действующей на окружности.

Tеорема 1.5 (см. [19], [17]). Для группы гомеоморфизмов окружности $G \subseteq$ Homeo $\left(\mathbb{S}^{1}\right)$ либо факторгруппа $G / H_{G}$ содержит свободную подгруппу с двумя образуюшими, либо существует вероятностная борелевская мера, инвариантная относительно группь $G$.

В работе Солодова [6] была сформулирована следующая альтернатива: для группы $G \subseteq$ Hотео $\left(\mathbb{S}^{1}\right)$ или группа $G$ содержит свободную подгруппу с двумя образующими, или $\operatorname{Fix} G^{S} \neq \varnothing$.

Так как по теореме 1.3 существование инвариантной меры эквивалентно условию Fix $G^{S} \neq \varnothing$, то альтернатива, сформулированная Солодовым, является слабой формой альтернативы из теоремы 1.5.

Приведем эквивалентную переформулировку теоремы 1.5, в которой все утверждения приводятся исключительно в терминах комбинаторных характеристик.

Tеорема 1.6 (см. [19], [17]). Для группы гомеоморфизмов окружности $G \subseteq$ Homeo $\left(\mathbb{S}^{1}\right)$ бакторгруппа $G / H_{G}$ либо содержит свободную подгруппу с двумя образующими, либо является коммутативной.

Для групп гомеоморфизмов окружности факторгруппа $G / H_{G}$ является универсальным инструментом при их классификации. Такая характеристика разбивает множество групп гомеоморфизмов окружности на два непересекающихся класса. В одном классе - группы с простейшими комбинаторными свойствами факторгруппы $G / H_{G}$, в другом - группы с максимально сложными комбинаторными свойствами факторгруппы $G / H_{G}$. K сожалению, данный классификационный инструмент неприменим к изучению самой канонической нормальной подгруппы $H_{G}$. Более детальное исследование нормальной подгруппы $H_{G}$ приводит к изучению индуцированного семейства групп гомеоморфизмов прямой (не окружности), а для них используемая характеристика неприменима. Поэтому задача формирования аналогичной характеристики для классификации групп гомеоморфизмов прямой является актуальной.

Для групп гомеоморфизмов прямой $G \subseteq \mathrm{Homeo}_{+}(\mathbb{R})$ в терминах комбинаторных характеристик также удается сформулировать критерий существования инвариантной меры. Очевидно, что в отличие от критерия существования инвариантной меры для группы гомеоморфизмов окружности условия на факторгруппу $G / H_{G}$ должны быть более жесткими.

ТЕОРема 1.7 (см. [19], [17]). Для группъ гомеоморфизмов прямой $G \subseteq$ Homeо $_{+}(\mathbb{R})$ с непустым минимальным множеством либо бакторгруппа $G / H_{G}$ содержит свободную подполугруппу с двумя образующими, либо существует 
борелевская мера, конечная на компактах и инвариантная относительно групnъ $G$.

Приведем эквивалентную переформулировку теоремы 1.7, в которой все утверждения приводятся исключительно в терминах комбинаторных характеристик.

Tеорема 1.8 (см. [19], [17]). Для группы гомеоморфизмов прямой $G \subseteq$ Homeо $_{+}(\mathbb{R})$ с непустым минимальным множеством факторгруппа $G / H_{G}$ либо содержит свободную подполугруппу с двумя образующими, либо является коммутативной.

Для групп гомеоморфизмов прямой свойства факторгруппы $G / H_{G}$ из теоремы 1.8 могут быть положены в основу классификационной схемы. Вместе с тем такая схема обладает рядом недостатков. Факторгруппа $G / H_{G}$, содержащая свободную подполугруппу с двумя образующими, может либо быть разрешимой (комбинаторно простая группа $G=\langle t+1,2 t\rangle$ ), либо быть группой со свободной подгруппой с двумя образующими (такая группа имеет максимальную комбинаторную сложность). Причина такого недостатка отражена в теореме 1.7. В силу отмеченной теоремы характеристика в виде факторгруппы $G / H_{G}$ разбивает множество всех групп гомеоморфизмов прямой на три класса: группы с инвариантной мерой; группы с непустым минимальным множеством и без инвариантной меры; группы с пустым минимальным множеством. Поэтому задача формулирования более приемлемой характеристики для классификации групп гомеоморфизмов прямой остается актуальной.

\section{3. Проективно инвариантные меры.}

ОПРЕДЕЛЕниЕ 1.4. Борелевская мера $\mu$, конечная на компактах, называется проективно инвариантной относительно группы $G \subseteq \mathrm{Homeo}_{+}(X)$, если для любого элемента $g \in G$ найдется $c_{g}>0$ такое, что для любого борелевского подмножества $B \subseteq \mathbb{R}$ справедливо условие $\mu\left(g^{-1}(B)\right)=c_{g} \mu(B)$.

Сформулируем важный критерий существования проективно инвариантной меры, использующий как топологические, так и алгебраические характеристики. Для любого элемента $q \in \mathrm{HomeO}_{+}(\mathbb{R})$ будем пользоваться обозначениями

$$
\begin{gathered}
T_{q}=\sup \{t: q(t)=t\}, t_{q}=\inf \{t: q(t)=t\}, \quad \text { если } \operatorname{Fix}\langle q\rangle \neq \varnothing ; \\
T_{q}=t_{q}=-\infty, \quad \text { если } \operatorname{Fix}\langle q\rangle=\varnothing .
\end{gathered}
$$

Определим еще одно каноническое подмножество группы $G \subseteq \mathrm{Homeo}_{+}(\mathbb{R})$

$$
C_{G}=\left(G \backslash G^{S}\right) \cup G_{\infty}^{S} .
$$

Tеорема 1.9 (см. [14], [17]). Пусть $G \subseteq \mathrm{Homeo}_{+}(\mathbb{R})$, для которой не существует борелевской инвариантной меры, конечной на компактах. Для существования борелевской меры, конечной на компактах и проективно инвариантной относительно группы $G$, необходимо и достаточно, чтобы одновременно выполнялись условия

1) множество $G_{\infty}^{S}$ является подгруппой и факторгруппа $G / G_{\infty}^{S}$ некоммутативная; 
2) для всякого $g \in G^{S} \backslash C_{G}$ справедливы следующие условия: $t_{g}, T_{g}$ конечны и для любых $t \in]-\infty, t_{g}[, T \in] T_{g},+\infty[$ выполнено соотношение $\operatorname{sign}[g(t)-t]=-\operatorname{sign}[g(T)-T]$

3) для любих $g_{1}, g_{2} \in G^{S} \backslash C_{G}$ либо $t_{g_{1}}=t_{g_{2}} u T_{g_{1}}=T_{g_{2}}$, либо $\left[t_{g_{1}}, T_{g_{1}}\right] \cap$ $\left[t_{g_{2}}, T_{g_{2}}\right]=\varnothing$.

Для групп, не имеющих инвариантной меры, мы можем сформулировать иной критерий существования проективно инвариантной меры, также использующий свойства канонически выделенных подмножеств $G_{\infty}^{S}$ и $C_{G}$ исходной группы $G \subseteq \mathrm{Homeo}_{+}(\mathbb{R})$.

TеOРема 1.10 (см. [15], [17]). Пусть для $G \subseteq$ Homeо $_{+}(\mathbb{R})$ не существует инвариантной борелевской меры, конечной на компактах. Для существования борелевской меры, конечной на компактах и проективно инвариантной относительно группы $G$, необходимо и достаточно, чтобы подмножества $G_{\infty}^{S}$ и $C_{G}$ являлисъ подгруппами и выполнялосъ условие $C_{G} \neq G_{\infty}^{S}$.

ЗАмЕчание 1.5 (см. [15], [17]). Если для группы $G \subseteq$ Homeo+ $_{+}(\mathbb{R})$ существует борелевская мера $\mu$, конечная на компактах и проективно инвариантная относительно группы $G$, но не существует инвариантной борелевской меры, конечной на компактах, то имеют место следующие утверждения: $G_{\infty}^{S}=H_{G}$; факторгруппы $G / C_{G}, C_{G} / G_{\infty}^{S}$ являются коммутативными, а факторгруппа $C_{G} / G_{\infty}^{S}$ нециклическая; мера $\mu$ для группы $C_{G}$ является инвариантной.

Для групп без инвариантной меры мы можем сформулировать еще один результат о существовании проективно инвариантной меры, основанный лишь на наличии специальной нормальной подгруппы.

Tеорема 1.11 (см. [16], [17]). Пусть для $G \subseteq$ Hотео $_{+}(\mathbb{R})$ не существует инвариантной борелевской меры, конечной на компактах. Для существования борелевской меры, конечной на компактах и проективно инвариантной относительно группы $G$, необходимо и достаточно, чтобы существовала нормальная подгруппа $\Gamma \subseteq G$ со следующими свойствами: для группи $\Gamma$ существует инвариантная мера; факторгруппа $\Gamma /\left\langle\Gamma^{S}\right\rangle$ нециклическая.

Достаточное условие из теоремы 1.11 было сформулировано в работе Plante [20] в иной эквивалентной форме. Формулировка была дана в терминах характера, построенного для группы $G$ по мере, инвариантной относительно нормальной подгруппы Г.

ЗАмЕЧАнИЕ 1.6. В силу замечаний 1.2 и 1.3 в теореме 1.11 для нормальной подгруппы $Г$ выполняется условие $\Gamma^{S}=\left\langle\Gamma^{S}\right\rangle$. Из замечания 1.1 следует, что подгруппа Г содержит свободно действующий элемент.

ЗАМЕчАНИЕ 1.7. В силу предложения 1.1 и теоремы 1.9 для группы $G \subseteq$ $\mathrm{Homeo}_{+}(\mathbb{R})$ из существования проективно инвариантной меры следует, что минимальное множество непусто.

Для специального класса групп гомеоморфизмов прямой сформулируем критерий существования проективно инвариантной меры в терминах комбинаторных характеристик. 
Tеорема 1.12 (см. [16], [17]). Пусть групna $G \subseteq \mathrm{Homeo}_{+}(\mathbb{R})$ содержит нормальную подгруппу Г с инвариантной мерой и свободно действующим элементом. Тогда либо факторгруппа $G / H_{G}$ содержст свободную подгруппу с двумя образующими, либо существует проективно инвариантная мера.

В работе Plante [20] было сформулировано достаточное условие существования проективно инвариантной меры, которое значительно более жесткое, чем в теореме 1.12: для группы $G \subseteq \mathrm{Homeo}_{+}(\mathbb{R})$, содержащей нормалъную подгруппу Г с инвариантной мерой и свободно действуюшим элементом, из аменабельности группы $G$ следует существование проективно инвариантной меры.

Проверка наличия нормальной подгруппы с теми или иными свойствами является весьма сложной процедурой. Теорема 1.12, в отличие от теоремы 1.11 , позволяет заменить проверку тяжелого условия о наличии нецикличной факторгруппы $\Gamma /\left\langle\Gamma^{S}\right\rangle$ для нормальной подгруппы $\Gamma$ на проверку условий о наличии только лишь свободно действующего элемента в нормальной подгруппе $Г$ и свободной подгруппы в факторгруппе $G / H_{G}$.

Приведем эквивалентную переформулировку теоремы 1.12 , в которой все утверждения приводятся исключительно в терминах комбинаторных характеристик.

TeOpema 1.13 (см. [16], [17]). Пусть групnа $G \subseteq \mathrm{Homeo+}_{+}(\mathbb{R})$ содержит нормалъную подгруппу Г с инвариантной мерой и свободно действующим элементом. Тогда либо факторгруппа $G / H_{G}$ содержит свободную подгруппу с двумя образующими, либо факторгруппа $G / H_{G}$ является разрешимой гpynпой.

\section{§ 2. Критерии существования инвариантной и проективно инвариантной мер в терминах коммутанта $[G, G]$}

Сформулируем критерий существования инвариантной меры в терминах коммутанта $[G, G]$.

Tеорема 2.1. Пусть $G \subseteq$ Нотео $_{+}(\mathbb{R})$ и минимальное множество групnъ $G$ непустое. Тогда для существования инвариантной борелевской меры, конечной на компактах, необходимо и достаточно, чтобы выполнялось условие

$$
[G, G] \subseteq H_{G}
$$

Доказательство. Необходимость. Пусть для группы $G$ существует инвариантная мера. По замечанию 1.3 имеет место равенство $H_{G}=G^{S}$, следовательно $\left\langle G^{S}\right\rangle=G^{S}$. Тогда по теореме 1.1 факторгруппа $G / H_{G}$ коммутативна, откуда и следует вложение (2.1).

Достаточность. Пусть минимальное множество дискретно. Тогда по теоремам 1.2 и 1.3 для группы $G$ существует инвариантная мера.

Пусть минимальное множество недискретно. Из условия (2.1) следует, что факторгруппа $G / H_{G}$ будет коммутативной. Факторгруппа $G / H_{G}$ может быть реализована как группа гомеоморфизмов на минимальном множестве $E(G)$. Действие левого класса смежности $[g]$ группы $G$ по нормальной подгруппе $H_{G}$ 
определяется по следующему правилу: $[g](t)=g(t)$ для любого $t \in E(G)$. Тогда из коммутативности факторгруппы $G / H_{G}$ следует, что любые два элемента $g_{1}, g_{2} \in G$ коммутируют на минимальном множестве $E(G)$, т.е. для любого $t \in E(G)$ справедливо правило перестановочности $g_{1} g_{2}(t)=g_{2} g_{1}(t)$. Заметим, что для любого элемента $g \in G^{S}$ найдется точка $\bar{t} \in E(G)$ такая, что $g(\bar{t})=\bar{t}$. В силу отмеченного условия перестановочности для каждого $q \in G$ справедлива цепочка соотношений $g(q(\bar{t}))=q(g(\bar{t}))=q(\bar{t})$.

Это означает, что вместе с точкой $\bar{t}$ точка $q(\bar{t})$ также принадлежит множеству Fix $g$. Так как это справедливо для любого элемента $q \in G$, то будет справедливо вложение

$$
E(G) \subseteq \operatorname{Fix} g, \quad g \in G^{S} .
$$

Но для такой группы $G$ будет выполняться условие $\mathrm{Fix} G^{S} \neq \varnothing$. Тогда по теореме 1.3 для такой группы $G$ существует инвариантная мера.

Теперь сформулируем критерий существования проективно инвариантной меры также в терминах коммутанта $[G, G]$.

Tеорема 2.2. Пусть $G \subseteq$ Нотео $_{+}(\mathbb{R})$ и минимальное множество группъ $G$ непустое. Для существования борелевской меры, конечной на компактах и проективно инвариантной относительно группы $G$, необходимо и достаточно, чтобы выполнялось вложение

$$
[G, G] \cap G^{S} \subseteq H_{G}
$$

ДокАЗАТЕЛЬство. Необходимость. Пусть существует проективно инвариантная мера.

Случай 1. Для группы $G$ существует инвариантная мера. По теореме 2.1 это эквивалентно условию $[G, G] \subseteq H_{G}$, откуда и следует вложение (2.3).

Случай 2. Для группы $G$ не существует инвариантной меры. По замечанию 1.5 факторгруппа $G / C_{G}$ коммутативна и поэтому $[G, G] \subseteq C_{G}$. По тому же замечанию 1.5 имеет место равенство $G_{\infty}^{S}=H_{G}$. В силу определения для группы $C_{G}$ выполняется условие $C_{G}^{S}=G_{\infty}^{S}$. Из полученных соотношений и следует вложение (2.3).

Достаточность. Пусть выполняется вложение (2.3).

Случай 1. Пусть выполняется условие

$$
[G, G] \subseteq H_{G}
$$

Тогда по теореме 2.1 для группы $G$ существует инвариантная мера.

Случай 2. Пусть выполняется условие

$$
[G, G] \backslash H_{G} \neq \varnothing .
$$

Для такой группы $G$ не существует инвариантной меры, и по теореме 1.2 минимальное множество группы $G$ недискретное.

Для коммутанта будем пользоваться обозначением $Q=[G, G]$. Очевидно, что $Q^{S}=Q \cap G^{S}$. Тогда из вложения (2.3) следует, что $Q^{S} \subseteq H_{G}$. Следовательно, имеет место цепочка соотношений $\left\langle Q^{S}\right\rangle=Q^{S}=Q_{\infty}^{S} \subseteq H_{G}$. Из свойства 
(2.5) вытекает, что $Q \neq\left\langle Q^{S}\right\rangle$. Тогда по замечанию 1.1 в группе $Q$ существует свободно действующий элемент, а по теореме 1.4 для группы $Q$ существует инвариантная мера. В таком случае по теореме 1.13 факторгруппа $G / H_{G}$ либо содержит свободную подгруппу с двумя образующими, либо является разрешимой, ступени не более двух.

Коммутант $Q$ нормален в группе $G$, и для любого $g \in G$ справедливо соотношение $g G^{S} g^{-1}=G^{S}$. Тогда из представления $\left\langle Q^{S}\right\rangle=Q \cap G^{S}$ следует, что $\left\langle Q^{S}\right\rangle$ - нормальная подгруппа группы $G$ и, соответственно, она нормальна в $H_{G}$. Имеет место изоморфизм

$$
\left(G /\left\langle Q^{S}\right\rangle\right) /\left(H_{G} /\left\langle Q^{S}\right\rangle\right) \cong G / H_{G}
$$

Факторгруппа $G / Q$ коммутативна по построению. По теореме 1.1 факторгруппа $Q / Q^{S}$ также коммутативна. Тогда в силу изоморфизма

$$
\left(G /\left\langle Q^{S}\right\rangle\right) /\left(Q /\left\langle Q^{S}\right\rangle\right) \cong G / Q
$$

факторгруппа $G /\left\langle Q^{S}\right\rangle$ будет разрешимой группой, ступени не более двух. В таком случае в силу изоморфизма (2.6) факторгруппа $G / H_{G}$ не может содержать свободной подгруппы с двумя образующими и по теореме 1.12 для группы $G$ существует проективно инвариантная мера.

Весьма интересен следующий вопрос. Можно ли условие (2.3) переформулировать в терминах локальных свойств графиков самих гомеоморфизмов из коммутанта $[G, G]$ либо исходной группы $G$ ?

ОПРЕДЕЛЕНИЕ 2.1. Пусть $G \subseteq \operatorname{Homeo}_{+}(\mathbb{R})$ - группа с непустым минимальным множеством. Элемент $g \in G$ называется плоским, если не существует тройки точек $t_{1}, t_{2}, t_{3}, t_{2} \in E(G)$ в случае недискретного минимального множества, либо тройки точек $t_{1}, t_{2}, t_{3}, t_{2} \in \mathrm{Fix} G^{s}$ в случае дискретного минимального множества, для которых выполняются условия

$$
t_{1}<t_{2}<t_{3}, \quad g\left(t_{1}\right)=t_{1}, \quad g\left(t_{2}\right) \neq t_{2}, \quad g\left(t_{3}\right)=t_{3} .
$$

\section{Справедлива}

Лемма 2.1. Пусть $G \subseteq$ Нотео $_{+}(\mathbb{R})$ - группа с непустым минимальным множеством. Для того чтобы выполнялось вложение

$$
[G, G] \cap G^{S} \subseteq H_{G}
$$

необходимо и достаточно, чтобы каждый элемент коммутанта $[G, G]$ был плоским.

ДокАЗАТЕЛЬСтво. Для коммутанта будем использовать обозначение $Q=$ $[G, G]$. Несложно заметить, что в случае конечности значения $T_{q}\left(t_{q}\right)$ для элемента $q \in G$ точка $T_{q}\left(t_{q}\right)$ всегда принадлежит минимальному множеству группы, т.е. $T_{q} \in E(G) \quad\left(t_{q} \in E(G)\right)$ в случае недискретности минимального множества и $T_{q} \in \operatorname{Fix} G^{s} \quad\left(t_{q} \in \operatorname{Fix} G^{s}\right)$ в случае дискретности минимального множества. 
Необходимость. Пусть выполняется вложение (2.9). Очевидно, что $Q^{S} \subseteq G^{S}$. Тогда из вложения (2.9) следует, что $Q^{S} \subseteq H_{G}$ и поэтому все элементы $Q^{S}$ являются плоскими. Каждый элемент $q \in Q \backslash Q^{S}$ свободно действующий и поэтому является плоским. Следовательно, все элементы коммутанта $Q$ плоские.

Достаточность. Предположим, что все элементы коммутанта плоские. Доказательство проведем от противного. Пусть нарушается условие (2.9), т.е. выполняется соотношение

$$
\left([G, G] \cap G^{S}\right) \backslash H_{G} \neq \varnothing .
$$

Шаг 1. Минимальное множество группы $G$ недискретное.

Действительно, из условия (2.10) следует условие $[G, G] \backslash H_{G} \neq \varnothing$. По теореме 2.1 для группы $G$ не существует инвариантной меры, и по замечанию 1.4 минимальное множество такой группы недискретное.

Шаг 2. Для любого элемента $q \in Q^{S}$ верно включение

$$
E(G) \cap\left(t_{q}, T_{q}\right) \subseteq \text { Fix } q .
$$

(В случае конечных $t_{q}$ или $T_{q}$ включение может быть усилено. Вместо интервала $\left(t_{q}, T_{q}\right)$ следует писать $\left[t_{q}, T_{q}\right),\left(t_{q}, T_{q}\right],\left[t_{q}, T_{q}\right]$ соответственно.)

Свойство (2.11) непосредственно следует из условия того, что все элементы группы $Q$ являются плоскими.

Шаг 3. Множество $Q^{S}$ инвариантно относительно операции сопряжения, т.е. $g Q^{S} g^{-1}=Q^{S}, g \in G$.

Это является следствием инвариантности множества $G^{S}$ относительно операции сопряжения, т.е. условия $g G^{S} g^{-1}=G^{S}, g \in G$, и нормальности подгруппы $Q$ группы $G$.

Шаг 4. Множество $Q_{\infty}^{S}$ является нормальной подгруппой группы $G$, и справедливо вложение $Q_{\infty}^{S} \subseteq H_{G}$.

Действительно, из условия (2.11) следует, что для каждого элемента $q \in Q_{\infty}^{S}$ выполняется включение $q \in H_{G}$, т.е. $Q_{\infty}^{S} \subseteq H_{G}$. Поэтому $Q_{\infty}^{S}$ является подгруппой. Так как $Q$ - нормальная подгруппа группы $G$, то множество $Q_{\infty}^{S}$ является инвариантным относительно операции сопряжения, т.е. $g Q_{\infty}^{S} g^{-1}=Q_{\infty}^{S}, g \in G$. Следовательно, подгруппа $Q_{\infty}^{S}$ будет нормальной подгруппой группы $G$.

Шаг 5. Для любого элемента $q \in Q^{S} \backslash Q_{\infty}^{S}$ значения $t_{q}, T_{q}$ конечны.

Очевидно, что для элемента $q$ одно из значений $t_{q}, T_{q}$ конечно. Для определенности пусть $T_{q}$ конечно и $q(t)>t, t \in\left(T_{q},+\infty\right)$. Предположим, что при этом $t_{q}=-\infty$. Так как минимальное множество $E(G)$ недискретно, то найдется элемент $g \in G$ такой, что $g\left(T_{q}\right) \neq T_{q}$. Для определенности будем считать, что $g\left(T_{q}\right)>T_{q}$. В противном случае такому условию будет удовлетворять обратный элемент. Рассмотрим элемент $\bar{q}=g q g^{-1}$. Из утверждений шагов 3 и 4 следует включение $\bar{q} \in Q^{S} \backslash Q_{\infty}^{S}$. Для такого элемента справедливы условия $T_{\bar{q}}=g\left(T_{q}\right)$, $t_{\bar{q}}=-\infty, \bar{q}(t)>t, t \in\left(T_{\bar{q}},+\infty\right)$. Так как $T_{q} \in E(G) \cap\left(-\infty, T_{\bar{q}}\right)$, то в силу шага 2 должно выполняться условие $\bar{q}\left(T_{q}\right)=T_{q}$. Так как $g\left(T_{q}\right)>T_{q}$, то для любого $k=1,2, \ldots$ найдется точка $\widehat{\tau}_{k}, T_{q} \leqslant \widehat{\tau}_{k}<T_{\bar{q}}$, для которой справедливы условия

$$
q\left(\widehat{\tau}_{k}\right)=\bar{q}^{k}\left(\widehat{\tau}_{k}\right), \quad q(t)>\bar{q}^{k}(t), \quad t \in\left(\widehat{\tau}_{k}, T_{\bar{q}}\right), \quad k=1,2, \ldots
$$


(В действительности здесь выполняется более сильное свойство, которое нам не понадобится. Так как все элементы группы $Q$ плоские, минимальное множество $E(G)$ недискретное и $\bar{q}^{k}(t)=t, t \in E(G) \cap\left(-\infty, T_{\bar{q}}\right]$, то имеет место равенство $\widehat{\tau}_{k}=T_{q}$.) Качественное поведение графиков рассматриваемых гомеоморфизмов и характерных для них точек отмечено на рис. 1. Чтобы не загромождать изображения, графики гомеоморфизмов вокруг диагонали вне выделенных характерных точек не выделены отдельно.

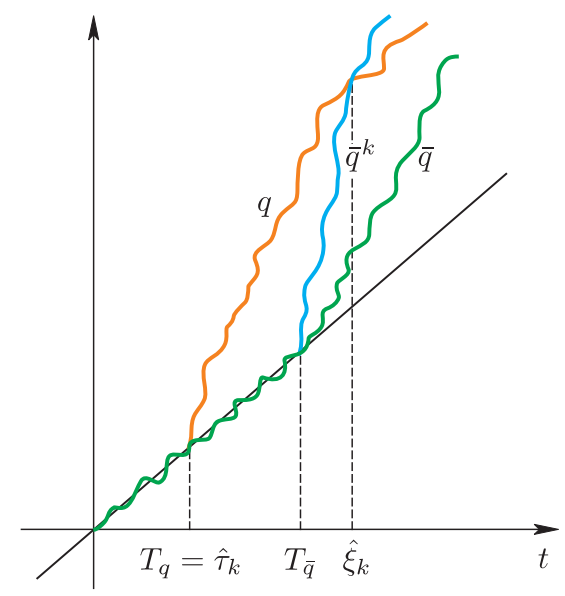

Рис. 1

Рассмотрим элемент $\bar{q}^{k}$ при достаточно большом фиксированном $k$. Для такого элемента найдется точка $\widehat{\xi}_{k}>T_{\bar{q}}$ такая, что

$$
q\left(\widehat{\tau}_{k}\right)=\bar{q}^{k}\left(\widehat{\tau}_{k}\right), \quad q\left(\widehat{\xi}_{k}\right)=\bar{q}^{k}\left(\widehat{\xi}_{k}\right), \quad q(t)>\bar{q}^{k}(t), \quad t \in\left(\widehat{\tau}_{k}, \widehat{\xi}_{k}\right), \quad k=1,2, \ldots
$$

Тогда для элемента $l=\bar{q}^{-k} q$ будет выполняться условие

$$
l\left(\widehat{\tau}_{k}\right)=\widehat{\tau}_{k}, \quad l\left(\widehat{\xi}_{k}\right)=\widehat{\xi}_{k}, \quad l(t)>t, \quad t \in\left(\widehat{\tau}_{k}, \widehat{\xi}_{k}\right) .
$$

Качественное поведение графика гомеоморфизма $l$ отмечено на рис. 2.

Так как $T_{\bar{q}} \in E(G)$ и $T_{\bar{q}} \in\left(\widehat{\tau}_{k}, \widehat{\xi}_{k}\right)$, то $\widehat{\tau}_{k}, \widehat{\xi}_{k}$ также принадлежат минимальному множеству $E(G)$. Поэтому элемент $l \in Q$ не может быть плоским. Противоречие. Следовательно значение $t_{q}$ также конечное.

Шаг 6. Для любых $q, \bar{q} \in Q^{S} \backslash Q_{\infty}^{S}$ либо $\left[t_{q}, T_{q}\right] \cap\left[t_{\bar{q}}, T_{\bar{q}}\right]=\varnothing$, либо $t_{q}=t_{\bar{q}}$, $T_{q}=T_{\bar{q}}$.

Доказательство проведем от противного. Для определенности пусть выполняются условия $t_{\bar{q}} \leqslant T_{q}<T_{\bar{q}}$. Также для определенности пусть для элементов $q, \bar{q}$ выполняются условия $q(t)>t, t>T_{q}, \bar{q}(t)>t, t>T_{\bar{q}}$. В противном случае этим условиям будут удовлетворять их обратные элементы. Качественное поведение графиков рассматриваемых гомеоморфизмов и характерных для них точек отмечено на рис. 3. Чтобы не загромождать изображения, графики гомеоморфизмов вокруг диагонали вне выделенных характерных точек не выделены отдельно. 


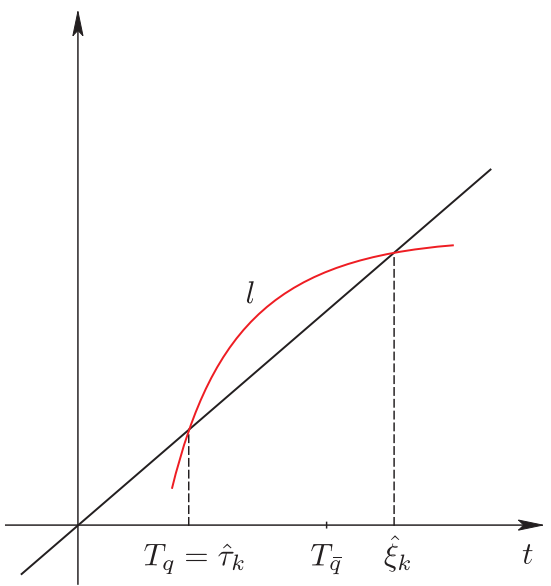

Рис. 2

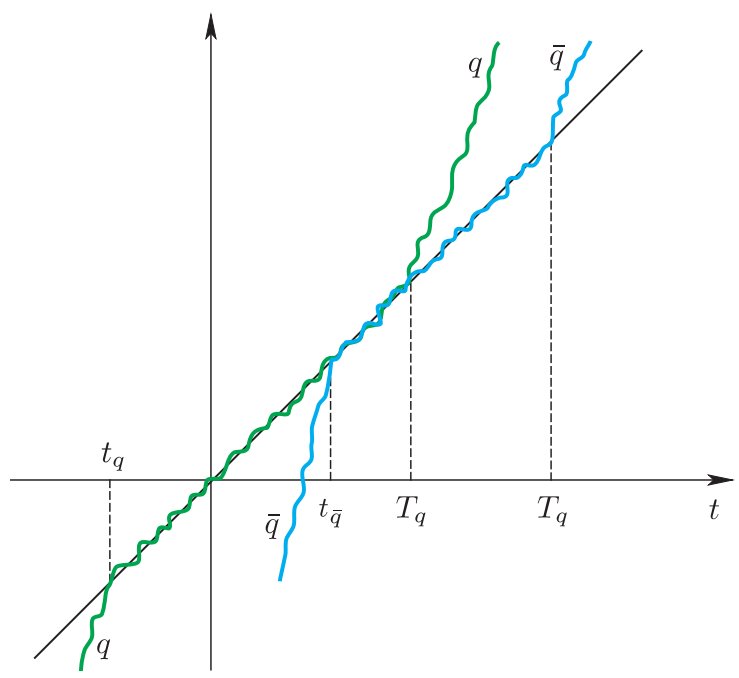

Рис. 3

Далее, буквально повторяя доказательство из шага 5 для элементов $q, \bar{q}$, придем в противоречие с тем, что элементы подгруппы $Q$ являются плоскими. Случай, когда $t_{\bar{q}}<T_{q} \leqslant T_{\bar{q}}$, изучается аналогичным образом. Следовательно, такое взаиморасположение элементов $q, \bar{q}$ невозможно.

Шаг 7. Для любого элемента $q \in Q^{S} \backslash Q_{\infty}^{S}$ справедливы условия

$$
\operatorname{sign}[q(t)-t]=-\operatorname{sign}[q(T)-T], \quad t \in\left(-\infty, t_{q}\right), \quad T \in\left(T_{q},+\infty\right) .
$$

Доказательство проведем от противного. Для определенности будем полагать, что $q(T)>T, T \in\left(T_{q},+\infty\right)$. В противном случае этому будет удовлетворять обратный элемент. Пусть условие (2.15) нарушается. Это означает, что 
для элемента $q$ справедливо условие $q(t)>t, t \in\left(-\infty, t_{q}\right)$. Так как минимальное множество $E(G)$ группы $G$ недискретное, то найдется элемент $g \in G$, для которого $g\left(T_{q}\right) \neq T_{q}$. Будем полагать, что $g\left(T_{q}\right)>T_{g}$. В противном случае этому свойству будет удовлетворять его обратный элемент. Рассмотрим элемент $\bar{q}=g q g^{-1}$. Очевидно, что $\bar{q} \in Q^{S} \backslash Q_{\infty}^{S}$ и $T_{\bar{q}}=g\left(T_{q}\right)$. В силу шага 6 будет выполняться условие $T_{q}<t_{\bar{q}}$. Заметим, что для элемента $\bar{q}$ также справедливо условие $\bar{q}(t)>t, t \in\left(-\infty, t_{\bar{q}}\right) \cup\left(T_{\bar{q}},+\infty\right)$. Для достаточно большого натурального $k$ рассмотрим элемент $q^{k}$. Для такого элемента найдутся точки $\widehat{\tau}_{k}, \widehat{\xi}_{k}$, $\widehat{\tau}_{k}<t_{q}, T_{q}<\widehat{\xi}_{k}<t_{\bar{q}}$, такие, что

$$
q^{k}\left(\widehat{\tau}_{k}\right)=\bar{q}\left(\widehat{\tau}_{k}\right), \quad q^{k}\left(\widehat{\xi}_{k}\right)=\bar{q}\left(\widehat{\xi}_{k}\right), \quad \bar{q}(t)>q^{k}(t), \quad t \in\left(\widehat{\tau}_{k}, \widehat{\xi}_{k}\right)
$$

Качественное поведение графиков рассматриваемых гомеоморфизмов и характерных для них точек отмечено на рис. 4. Чтобы не загромождать изображения, графики гомеоморфизмов вокруг диагонали вне выделенных характерных точек не выделены отдельно.

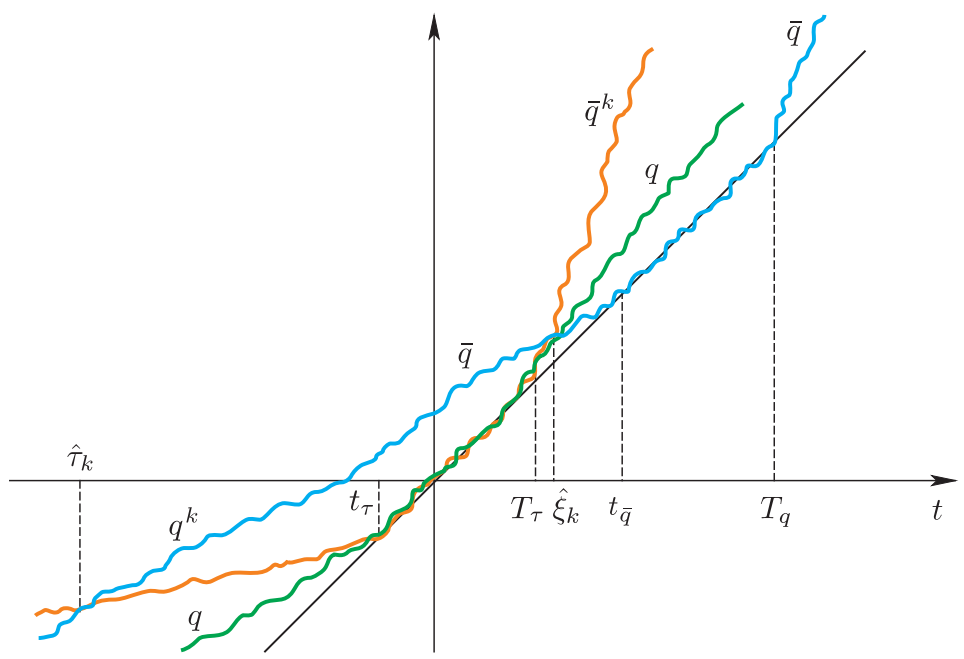

Рис. 4

Образуем элемент $l=q^{-k} \bar{q}$. Для него будут выполняться условия

$$
l\left(\widehat{\tau}_{k}\right)=\widehat{\tau}_{k}, \quad l\left(\widehat{\xi}_{k}\right)=\widehat{\xi}_{k}, \quad l(t)>t, \quad t \in\left(\widehat{\tau}_{k}, \widehat{\xi}_{k}\right) .
$$

Так как $t_{q}, T_{q} \in E(G) \cap\left(\widehat{\tau}_{k}, \widehat{\xi}_{k}\right)$, то точки $\widehat{\tau}_{k}, \widehat{\xi}_{k}$ также принадлежат минимальному множеству $E(G)$. В таком случае элемент $l \in Q$ не может быть плоским. Следовательно, для элементов $q \in Q^{S} \backslash Q_{\infty}^{S}$ выполняется условие (2.15).

Шаг 8. Факторгруппа $Q / Q_{\infty}^{S}$ некоммутативная.

Из условия (2.10) и условия $Q_{\infty}^{S} \subseteq H_{G}$ (см. шаг 3) следует существование элемента $\widetilde{q}$, удовлетворяющего включению $\widetilde{q} \in Q^{S} \backslash Q_{\infty}^{S}$. Для такого элемента значения $t_{\widetilde{q}}, T_{\widetilde{q}}$ конечны (шаг 5 ). Как это отмечалось и в предыдущих шагах, в силу недискретности минимального множества $E(G)$ существует элемент $g \in G$ со свойством $g\left(T_{\widetilde{q}}\right)>T_{\widetilde{q}}$. Образуем элемент $\bar{q}=g \widetilde{q} g^{-1}$. Очевидно, 
что $\bar{q} \in Q^{S} \backslash Q_{\infty}^{S}$ и выполняются равенства $t_{\bar{q}}=g\left(t_{\widetilde{q}}\right), T_{\bar{q}}=g\left(T_{\widetilde{q}}\right)$, а в силу шага 6 получим $t_{\bar{q}}>T_{\widetilde{q}}$. Так как $Q_{\infty}^{S} \subseteq H_{G}$, то факторгруппу $Q / Q_{\infty}^{S}$ можем реализовать как группу, действующую на минимальном множестве $E(G)$. Для любого класса смежности $[q]$ группы $Q$ по подгруппе $Q_{\infty}^{S}$ и любого $t \in E(G)$ положим $[q](t)=q(t)$. Очевидно, что два построенных класса смежности $[\widetilde{q}],[\bar{q}]$ различны. Более того, их действия на минимальном множестве $E(G)$ не коммутируют. Следовательно, факторгруппа $Q / Q_{\infty}^{S}$ некоммутативна.

Шаг 9. Для группы $Q$ не существует инвариантной меры.

На шаге 8 мы отметили, что в силу условия (2.10) в группе $Q$ существует элемент $\widetilde{q} \in Q^{S} \backslash Q_{\infty}^{S}$. Там же по элементу $\widetilde{q}$ был построен элемент $\bar{q} \in Q^{S} \backslash Q_{\infty}^{S}$. Для этих элементов справедливо условие $\left[t_{\widetilde{q}}, T_{\widetilde{q}}\right] \cap\left[t_{\bar{q}}, T_{\bar{q}}\right]=\varnothing$ и поэтому $\operatorname{Fix} Q=\varnothing$. По теореме 1.3 для такой группы не существует инвариантной меры.

Шаг 10. Для группы $Q$ существует проективно инвариантная мера.

Действительно, в силу шагов 4-8 группа $Q$ удовлетворяет всем условиям теоремы 1.9 , откуда и следует существование проективно инвариантной меры.

Шаг 11. Существование проективно инвариантной меры для группы $Q$ противоречит предположению (2.10).

Действительно, на шаге 4 мы уже отмечали, что $Q_{\infty}^{S}$ является нормальной подгруппой группы $G$. Для любого свободно действующего элемента $\bar{g} \in G$ сопряженные к нему элементы $g \bar{g} g^{-1}$ также являются свободно действующими. Для группы $Q$ существует проективно инвариантная мера $\mu$, и по замечанию 1.5 множество $C_{Q}$ является подгруппой группы $Q$ (нормальной подгруппой). Так как $C_{Q} \subseteq Q$ и $C_{a}$ состоит либо из элементов $Q_{\infty}^{S}$, либо из свободно действующих элементов, а $Q$ - нормальная подгруппа группы $G$, то $C_{Q}$ будет нормальной подгруппой группы $G$. Так как для группы $Q$ существует проективно инвариантная мера $\mu$, но не существует инвариантной меры, то по замечанию 1.5 мера $\mu$ будет инвариантной относительно подгруппы $C_{Q}$, а факторгруппа $C_{Q} / Q_{\infty}^{S}$ - нециклической. Тогда по теореме 1.11 для группы $G$ существует проективно инвариантная мера $\widetilde{\mu}$.

Так как для подгруппы $Q$ не существует инвариантной меры, то инвариантной меры не существует и для исходной группы $G$. Тогда по замечанию 1.5 проективно инвариантная мера $\widetilde{\mu}$ будет инвариантной на подгруппе $C_{G}$. По тому же замечанию 1.5 факторгруппа $G / C_{G}$ будет коммутативной и поэтому будет справедливым вложение $Q=[G, G] \subseteq C_{G}$. Следовательно, мера $\widetilde{\mu}$ будет инвариантной и на $Q$. Но на шаге 9 мы показали, что для группы $Q$ не существует инвариантной меры, - противоречие. Следовательно, условие $(2.10)$ не может выполняться. Лемма доказана.

Лемму 2.1 мы можем несколько переформулировать. Вместо коммутанта $[G, G]$ будет фигурировать исходная группа $G$.

Лемма 2.2. Пусть $G \subseteq$ Homeо $_{+}(\mathbb{R})$ - группа с непустым минимальным множеством. Для того чтобы выполнялось вложение

$$
[G, G] \cap G^{S} \subseteq H_{G}
$$

необходимо и достаточно, чтобы каждый элемент группы $G$ был плоским. 
ДокАЗАТЕЛЬСтво. Достаточность очевидна и непосредственно следует из леммы 2.1.

Необходимость. Пусть для группы $G$ существует проективно инвариантная мера, что в силу теоремы 2.2 эквивалентно условию (2.18).

Случай 1 . Для группы $G$ существует инвариантная мера. Тогда по теореме 1.3 выполняется условие $\operatorname{Fix} G^{S} \neq \varnothing$, а из определения множества Fix $G^{S}$ следует, что для любого элемента $g \in G^{S}$ выполняется вложение

$$
\text { Fix } G^{S} \subseteq \operatorname{Fix} g \text {. }
$$

Если минимальное множество дискретное, то из (2.19) будет следовать, что всякий элемент группы $G$ является плоским. Пусть минимальное множество недискретное. Из определения минимального множества следует вложение $E(G) \subseteq \operatorname{Fix} G^{S}$. Тогда из (2.19) вытекает, что всякий элемент группы $G$ является плоским.

Случай 2. Для группы $G$ не существует инвариантной меры. По замечанию 1.5 для такой группы справедливо равенство $G_{\infty}^{S}=H_{G}$. Поэтому любой элемент $g \in G_{\infty}^{S}$ является плоским. Остается показать, что всякий элемент $g \in G^{S} \backslash G_{\infty}^{S}$ также является плоским. Так как $G^{S} \backslash G_{\infty}^{S}=G \backslash C_{G}$, то по теореме 1.9 для элемента $g \in G^{S} \backslash G_{\infty}^{S}$ значения $t_{g}, T_{g}$ конечны. Очевидно, что точки $t_{g}, T_{g}$ принадлежат минимальному множеству, т.е. $t_{g}, T_{g} \in E(G)$. Если покажем, что не существует точки $\widehat{t} \in\left(t_{g}, T_{g}\right) \cap E(G)$, то лемма будет доказана.

Докажем от противного. Пусть существует точка $\widehat{t} \in\left(t_{g}, T_{g}\right) \cap E(G)$. Тогда найдется элемент $\widehat{g} \in G$, для которого точка $\widehat{g}\left(T_{g}\right)$ окажется в достаточно малой окрестности точки $\widehat{t}$ и, в частности, выполняется условие $\widehat{g}\left(T_{g}\right) \in\left(t_{g}, T_{g}\right)$. Рассмотрим элемент $l=\widehat{g} g \widehat{g}^{-1}$. Для такого элемента точки $t_{l}, T_{l}$ также конечны и выполняется равенство $T_{l}=\widehat{g}\left(T_{g}\right)$. Но это противоречит условию 3$)$ теоремы 1.9. Следовательно, точки $\widehat{t} \in\left(t_{g}, T_{g}\right) \cap E(G)$ не существует. Лемма доказана.

\section{§ 3. О специальной подгруппе с двумя образующими в группе гомеоморфизмов прямой со свободно действующим элементом и без проективно инвариантной меры}

В теоремах 1.11, 1.12 сформулированы критерии существования проективно инвариантной меры. Такие критерии сформулированы для групп, содержащих нормальную подгруппу с инвариантной мерой и свободно действующим элементом. Используя теорему 2.2 и лемму 2.2 , мы можем получить критерий существования проективно инвариантной меры для более широкого класса групп, т.е. для групп, только лишь содержащих свободно действующий элемент. Будет показано, что для группы со свободно действующим элементом критерием существования проективно инвариантной меры является отсутствие некоторой специальной подгруппы с двумя образующими.

Теорема 3.1. Пусть $G \subseteq \mathrm{Homeo}_{+}(\mathbb{R})$ - группа со свободно действующим элементом $\bar{g} \in G, \operatorname{Fix} \bar{g}=\varnothing$. Для существования проективно инвариантной 
меры необходимо и достаточно, чтобы в группе $G$ отсутствовала подгруппа $\Lambda \subseteq G, \Lambda=\langle p, q\rangle$, в которой элемент $p$ свободно действующий и для элементов $p, q$ существуют точки $t_{0}, t_{1} \in E(G), t_{0}<t_{1}$, со следующими свойствами (puc. 5):

$$
\begin{array}{cl}
p(t)>t, \quad t \in \mathbb{R}, \quad & p\left(t_{0}\right) \in\left(t_{0}, t_{1}\right) ; \\
q\left(t_{0}\right)=t_{0}, \quad q\left(t_{1}\right)=t_{1}, \quad & q(t)>t, \quad t \in\left(t_{0}, t_{1}\right) .
\end{array}
$$

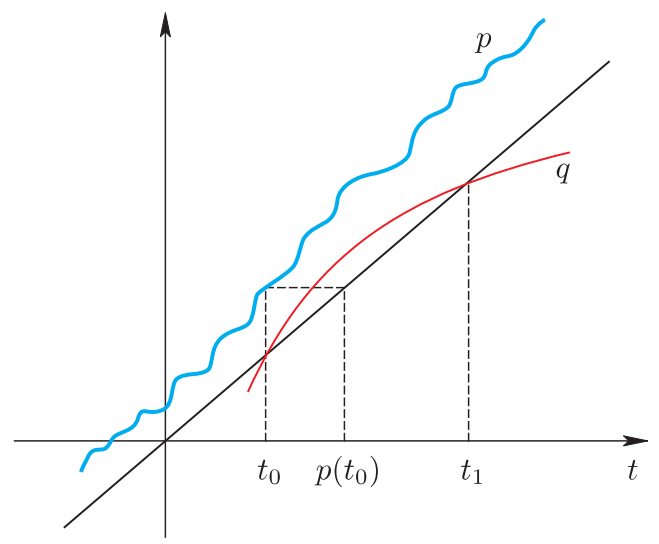

Рис. 5

ДокАЗАТЕЛЬство. Необходимость. Пусть для группы $G$ существует проективно инвариантная мера. Доказательство проведем от противного. Предположим, что в группе $G$ существует подгруппа $\Lambda=\langle p, q\rangle$ из теоремы. Так как $p$ - свободно действующий элемент, то по предложению 1.1 минимальное множество подгруппы $\Lambda$ непусто. Очевидно, что для группы $\Lambda$ множество $\Lambda^{S}$ не образует группы, т.е. $\Lambda^{S} \neq\left\langle\Lambda^{S}\right\rangle$. Тогда по замечанию 1.3 для группы $\Lambda$ не существует инвариантной меры. В таком случае по теореме 1.3 имеет место условие Fix $\Lambda^{S}=\varnothing$ и в силу теоремы 1.2 минимальное множество недискретное. Из условия $p\left(t_{0}\right) \in\left(t_{0}, t_{1}\right)$ следует, что $E(\Lambda) \cap\left(t_{0}, t_{1}\right) \neq \varnothing$. Тогда из свойств элемента $q$ на интервале $\left[t_{0}, t_{1}\right]$ будет вытекать включение $t_{0}, t_{1} \in E(\Lambda)$. Следовательно, элемент $q$ в подгруппе $\Lambda$ не является плоским. Тогда по теореме 2.2 и лемме 2.2 для подгруппы $\Lambda$ не существует проективно инвариантной меры. Противоречие. Следовательно, в группе $G$ отсутствует подгруппа $\Lambda=\langle p, q\rangle$ из теоремы.

Достаточность. Пусть в группе $G$ отсутствует какая-либо подгруппа $\Lambda=$ $\langle p, q\rangle$ со свойствами из теоремы. Доказательство проведем от противного. Предположим, что для группы $G$ отсутствует проективно инвариантная мера. Из существования свободно действующего элемента и предложения 1.1 следует, что минимальное множество непусто. Так как для группы $G$ не существует проективно инвариантной меры, то по замечанию 1.4 минимальное множество недискретно. Более того, по теореме 2.2 и лемме 2.2 найдутся элемент $\widetilde{g}$ и 
тройка точек $\widetilde{t}_{1}, \widetilde{t}_{2}, \widetilde{t}_{3}$ со свойствами

$$
\begin{gathered}
\tilde{t}_{1}, \widetilde{t}_{2}, \widetilde{t}_{3} \in E(G), \quad \widetilde{t}_{1}<\widetilde{t}_{2}<\widetilde{t}_{3}, \\
\widetilde{g}\left(\tilde{t}_{1}\right)=\widetilde{t}_{1}, \quad \widetilde{g}\left(\widetilde{t}_{3}\right)=\widetilde{t}_{3}, \quad \widetilde{g}(t)>t, \quad t \in\left(\widetilde{t}_{1}, \widetilde{t}_{3}\right) .
\end{gathered}
$$

Для определенности будем считать, что $\bar{g}(t)>t, t \in \mathbb{R}$. Если $\bar{g}\left(\widetilde{t}_{1}\right) \in\left(\widetilde{t}_{1}, \widetilde{t}_{3}\right)$, то группа $\Lambda$ построена. Для этого достаточно положить $p=\bar{g}, q=\widetilde{g}, t_{0}=\widetilde{t}_{1}$, $t_{1}=\widetilde{t}_{3}$.

Пусть выполняется условие $\bar{g}\left(\widetilde{t_{1}}\right)>\widetilde{t}_{3}$. Далее нам следует рассмотреть два случая.

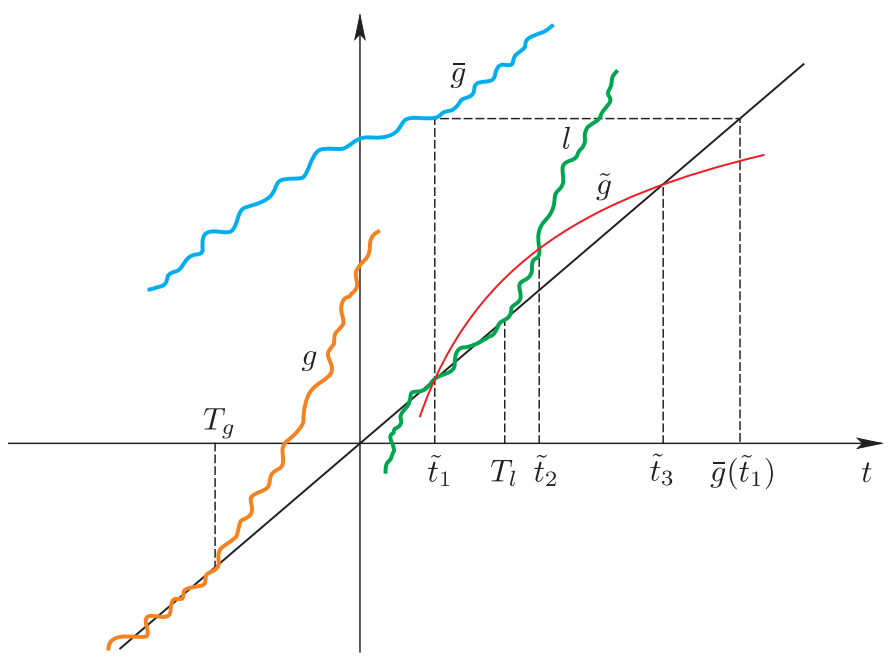

Рис. 6

Случай 1: $G^{S} \backslash G_{\infty}^{S} \neq \varnothing$. Выберем элемент $g \in G^{S} \backslash G_{\infty}^{S}$. Для элемента $g$ одно из значений $t_{g}, T_{g}$ обязательно конечно. Для определенности будем полагать, что значение $T_{g}$ конечно и $g(t)>t, t \in\left(T_{g},+\infty\right)$ (рис. 6). Очевидно, что точка $T_{g}$ принадлежит минимальному множеству, т.е. $T_{g} \in E(G)$. Так как $\widetilde{t}_{2} \in E(G) \cap\left(\widetilde{t}_{1}, \widetilde{t}_{3}\right)$, то из определения минимального множества следует существование элемента $\xi \in G$, для которого справедливо включение $\xi\left(T_{g}\right) \in\left(\widetilde{t}_{1}, \widetilde{t}_{3}\right)$. Рассмотрим элемент $l=\xi g \xi^{-1}$. Очевидно, что $l \in G^{S} \backslash G_{\infty}^{S}, T_{l}=\xi\left(T_{g}\right), l(t)>t$, $t \in\left(T_{l},+\infty\right)$.

Образуем элемент $\widehat{g}=l^{k} \widetilde{g} l^{-k}, k \in \mathbb{Z}^{+}$. Очевидно, что для элемента $\widehat{g}$ выполняются условия

$$
\begin{gathered}
\widehat{g}\left(l^{k}\left(\widetilde{t}_{1}\right)\right)=l^{k}\left(\widetilde{t}_{1}\right), \quad \widehat{g}\left(l^{k}\left(\widetilde{t}_{3}\right)\right)=l^{k}\left(\widetilde{t}_{3}\right), \\
l^{k}\left(\widetilde{t_{1}}\right)<T_{l}, \quad \widehat{g}(t)>t, \quad t \in\left(l^{k}\left(\widetilde{t}_{1}\right), l^{k}\left(\widetilde{t}_{3}\right)\right) .
\end{gathered}
$$

За счет выбора $k \in \mathbb{Z}^{+}$величину $\left[l^{k}\left(\widetilde{t}_{3}\right)-T_{l}\right]$ можно сделать сколь угодно большой так, чтобы выполнялось условие $\bar{g}\left(l^{k}\left(\widetilde{t}_{1}\right)\right) \in\left(l^{k}\left(\widetilde{t}_{1}\right), l^{k}\left(\widetilde{t}_{3}\right)\right)$ (рис. 7$)$. 


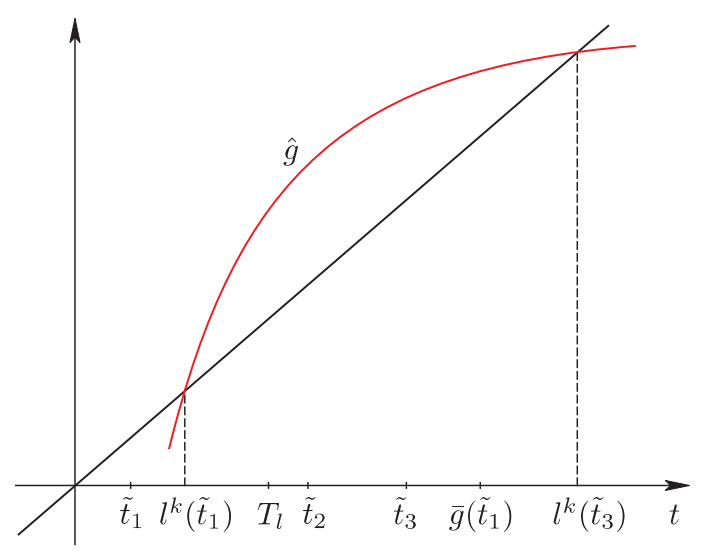

Рис. 7

Остается положить $p=\bar{g}, q=\widehat{g}, t_{0}=l^{k}\left(\widetilde{t}_{1}\right), t_{1}=l^{k}\left(\widetilde{t}_{3}\right)$ и группа $\Lambda$ построена. Случай 2: $G^{S} \backslash G_{\infty}^{S}=\varnothing, G^{S}=G_{\infty}^{S}$. Для такой группы $G$ имеет место равенство $G=C_{G}$, т.е. группа состоит из объединения множества $G_{\infty}^{S}$ и множества всех свободно действующих элементов. Более того, для такой группы $G$ множество $G_{\infty}^{S}$ не образует подгруппы. Действительно, если бы выполнялось условие $G_{\infty}^{S}=\left\langle G_{\infty}^{S}\right\rangle$, то в силу наличия свободно действующего элемента $\bar{g} \in G$ факторгруппа $G / G_{\infty}^{S}$ была бы нетривиальной. Тогда по теореме 1.4 для такой группы $G$ существует инвариантная мера, что противоречит нашим предположениям. Следовательно, выполняется условие $G_{\infty}^{S} \neq\left\langle G_{\infty}^{S}\right\rangle$. Тогда найдутся элементы $g, g_{1} \in G_{\infty}^{S}$ такие, что элемент $\breve{g}=g g_{1}$ будет свободно действующим. Для определенности будем полагать, что $\breve{g}(t)>t, t \in \mathbb{R}$. Найдется интервал $\left[t_{0}, t_{1}\right]$, для которого выполняются условия $g\left(t_{0}\right)=t_{0}, g\left(t_{1}\right)=t_{1}, g(t)>t$, $t \in\left(t_{0}, t_{1}\right)$, а также существует точка $\widehat{t} \in\left(t_{0}, t_{1}\right)$ со свойством $g_{1}(\widehat{t})=\widehat{t}$. Тогда для такого интервала будет справедливо и условие $\breve{g}\left(t_{0}\right) \in\left(t_{0}, t_{1}\right)$. Остается положить $p=\breve{g}, q=g$ и группа $\Lambda$ построена. Во всех рассмотренных случаях мы пришли к противоречию, показав, что из отсутствия проективно инвариантной меры следует существование группы $\Lambda$. Значит, для группы $G$ существует проективно инвариантная мера. Теорема доказана.

На основе рассуждений, проведенных в последнем абзаце доказательства теоремы 3.1, сформулируем

ЗАмечАниЕ 3.1. Для группы $\Lambda$ из теоремы 3.1 минимальное множество недискретно и содержит точки $t_{0}, t_{1}$, т.е. $t_{0}, t_{1} \in E(\Lambda)$.

Проверка наличия нормальной подгруппы с теми или иными свойствами является весьма сложной процедурой. Теорема 3.1, в отличие от теоремы 1.12 , позволяет заменить проверку тяжелых условий о наличии нормальной подгруппы со свободно действующим элементом и свободной подгруппы в факторгруппе $G / H_{G}$ на проверку условий о наличии только лишь свободно действующего элемента и специальной подгруппы $\Lambda$ в исходной группе $G$. 
Автор признателен всем участникам семинара "Динамические системы и эргодическая теория" и его руководителям Д. В. Аносову, А.М. Степину и Р. И. Григорчуку за полезные советы и внимание к работе.

\section{Список литературы}

[1] С. П. Новиков, “Топология слоений”, Тр. ММО, 14, Изд-во Моск. ун-та, М., 1965, 248-278; англ. пер.: S. P. Novikov, "Topology of foliations", Trans. Moscow Math. Soc., 14, Amer. Math. Soc., Providence, RI, 1965, 268-304.

[2] Л. Альфорс, Лекиии по квазиконформным отображениям, Мир, М., 1969, 133 с.; пер. с англ.: L. V. Ahlfors, Lectures on quasiconformal mappings, Van Nostrand Mathematical Studies, 10, D. Van Nostrand Co., Inc., Toronto, Ont.-New York-London, 1966, v+146 pp.

[3] Л. Альфорс, Преобразования Мёбиуса в многомерном пространстве, Мир, М., 1986, 112 с.; пер. с англ.: L.V. Ahlfors, Möbius transformations in several dimensions, Ordway Professorship Lectures Math., Univ. Minnesota, School of Mathematics, Minneapolis, Minn., 1981, ii+150 pp.

[4] M. M. Day, "Amenable semigroups", Illinois J. Math., 1:4 (1957), 509-544.

[5] Ф. Гринлиф, Инвариантные средние на топологических группах и их приложения, Мир, М., 1973, 136 с.; пер. с англ.: F. P. Greenleaf, Invariant means on topological groups and their applications, Van Nostrand Mathematical Studies, 16, Van Nostrand Reinhold Co., New York-Toronto, Ont.-London, 1969, ix+113 pp.

[6] В. В. Солодов, "Гомеоморфизмы окружности и слоения", Изв. АН СССР. Сер. матем., 48:3 (1984), 599-613; англ. пер.: V. V. Solodov, "Homeomorphisms of the circle and a foliation", Math. USSR-Izv., 24:3 (1985), 553-566.

[7] Л. А. Бекларян, "Вариационная задача с запаздывающим аргументом и ее связь с некоторой полугруппой отображений отрезка в себя", Докл. АH CCCP, 271:5 (1983), 1036-1040; англ. пер.: L. A. Beklaryan, "A variational problem with retarded argument and its relation to some semigroup of mappings of a segment into itself", Soviet Math. Dokl., 28:1 (1983), 1036-1040.

[8] Л. А. Бекларян, "Задача оптимального управления для систем с отклоняющимся аргументом и ее связь с конечно порожденной группой гомеоморфизмов $\mathbb{R}$, порожденной функциями отклонения аргумента", Докл. АН СССР, 317:6 (1991), 1289-1294; англ. пер.: L. A. Beklaryan, "An optimal control problem for systems with deviating argument and its connection with the finitely generated group of homeomorphisms of $R$ generated by deviation functions", Soviet Math. Dokl., 43:2 (1991), 600-605.

[9] Л.А. Бекларян, "О критерии топологической сопряженности квазисимметрической группы группе аффинных преобразований $\mathbb{R}^{\prime \prime}$ Матем. сб., 191:6 (2000), 31-42; англ. пер.: L. A. Beklaryan, "On a criterion for the topological conjugacy of a quasisymmetric group to a group of affine transformations of $\mathbb{R}$ ", Sb. Math., 191:6 (2000), 809-819.

[10] L. A. Beklaryan, "About canonical types of the differential equations with deviating argument", Funct. Differ. Equ., 8:1-2 (2001), 25-33.

[11] Л.А. Бекларян, Введение в теорию функиинально-дифференциальных уравнений. Групповой подход, Факториал Пресс, М., 2007, 288 с.

[12] Ю.И. Карлович, " $C^{*}$-алгебра операторов типа свертки с дискретными группами сдвигов и осциллирующими коэффициентами", Докл. АН CCCP, 302:3 (1988), 535-540; англ. пер.: Yu. I. Karlovich, " $C^{*}$-algebras of operators of convolution type with discrete groups of shifts and with oscillating coefficients", Soviet Math. Dokl., 38:2 (1989), 301-307. 
[13] Л. А. Бекларян, "K вопросу о классификации групп гомеоморфизмов $\mathbb{R}$, сохраняющих ориентацию. І. Инвариантные меры”, Матем. сб., 187:3 (1996), 23-54; англ. пер.: L. A. Beklaryan, "On the classification of groups of orientation-preserving homeomorphisms of $\mathbb{R}$. I. Invariant measures", Sb. Math., 187:3 (1996), 335-364.

[14] Л.А. Бекларян, "Критерий существования проективно инвариантной меры для групп гомеоморфизмов $\mathbb{R}$, сохраняющих ориентацию, связанный со структурой множества неподвижных точек", УМН, 51:3(309) (1996), 179-180; англ. пер.: L. A. Beklaryan, "A criterion connected with the structure of the fixed-point set for the existence of a projectively invariant measure for groups of orientation-preserving homeomorphisms of $\mathbb{R}^{\prime \prime}$ Russian Math. Surveys, 51:3 (1996), 539-540.

[15] Л.А. Бекларян, "K вопросу о классификации групп гомеоморфизмов $\mathbb{R}$, сохраняющих ориентацию. ІІ. Проективно инвариантные меры”, Матем. сб., 187:4 (1996), 3-28; англ. пер.: L. A. Beklaryan, "On the classification of groups of orientation-preserving homeomorphisms of $\mathbb{R}$. II. Projectively-invariant measures", Sb. Math., 187:4 (1996), 469-494.

[16] Л. А. Бекларян, "K вопросу о классификации групп гомеоморфизмов $\mathbb{R}$, сохраняющих ориентацию. III. $\omega$-проективно инвариантные меры", Матем. сб., 190:4 (1999), 43-62; англ. пер.: L. A. Beklaryan, "On the classification of groups of orientation-preserving homeomorphisms of $\mathbb{R}$. III. $\omega$-projectively invariant measures", Sb. Math., 190:4 (1999), 521-538.

[17] Л.А. Бекларян, "Группы гомеоморфизмов прямой и окружности. Топологические характеристики и метрические инварианты", УМН, 59:4(358) (2004), 3-68; англ. пер.: L. A. Beklaryan, "Groups of homeomorphisms of the line and the circle. Topological characteristics and metric invariants", Russian Math. Surveys, 59:4 (2004), 599-660.

[18] J. F. Plante, "Foliations with measure preserving holonomy", Ann. of Math. (2), 102:2 (1975), 327-361.

[19] Л.А. Бекларян, "Об аналогах альтернативы Титса для групп гомеоморфизмов окружности и прямой”, Матем. заметки, 71:3 (2002), 334-347; англ. пер.: L. A. Beklaryan, "On analogs of the Tits alternative for groups of homeomorphisms of the circle and of the line", Math. Notes, 71:3 (2002), 305-315.

[20] J. F. Plante, "Solvable groups acting on the line", Trans. Amer. Math. Soc., 278:1 (1983), 401-414.

\section{Лева Андреевич Бекларян}

(Leva A. Beklaryan)

Центральный экономико-математический институт

РАН, г. Москва

E-mail: beklar@cemi.rssi.ru
Поступила в редакцию 21.11.2013 и 03.10 .2014 